\title{
Knowledge about antibiotics and attitudes towards vaccination: Regression analysis of Wellcome Monitor survey data
}

\author{
Alistair Anderson \\ School of Sociology and Social Policy - University of Nottingham \\ School of Geographical Sciences - University of Bristol
}

\section{Abstract:}

Objectives:

To analyse associations within the UK public between vaccine-related attitudes and knowledge about the role of antibiotics for treating various infections.

\section{Design:}

Secondary analysis of a random-probability mixed-mode survey conducted by NatCen Social Research for the Wellcome Trust in late 2018.

\section{Setting:}

England, Wales, Scotland.

\section{Participants:}

2,708 members of the public recruited to the NatCen Panel from the British Social Attitudes randomprobability survey.

\section{Main outcome measures:}

Ordinal logistic regression models of antibiotic-related knowledge predicted by vaccine attitudes, controlling for age, sex, education, employment status, geography, health information habits, perceptions of individual agency, and interview mode. The outcome variable was based on a question about the kinds of infections antibiotics are useful for treating. Vaccine-attitude outcomes were perceptions of the risk of serious side-effects from vaccination and perceptions of the efficacy of vaccination as a preventative intervention.

\section{Results:}

Compared to the modal response category of 'Fairly low', respondents who rated the risk of serious side-effects from vaccination as 'Very high' $(\mathrm{OR}=3.34,95 \% \mathrm{CL}=2.13-5.25)$ or 'Fairly high' $(\mathrm{OR}=1.55,95 \% \mathrm{CL}=1.26-1.90)$ were more likely to have provided incorrect responses to questions about the utility of antibiotics for treating different types of infection. Conversely, respondents who felt that there was 'No risk at all' $(\mathrm{OR}=0.64,95 \% \mathrm{CL}=0.46-0.87)$ were less likely to have provided incorrect responses about the utility of antibiotics. Compared to the modal category of 'Almost always effective', respondents who felt that vaccines were 'Sometimes effective' ( $O R=1.25,95 \% \mathrm{CL}=1.05$ 1.49 ) as a preventative intervention were slightly more likely to have provided incorrect responses regarding antibiotics' utility. However, there were no other significant associations between perceptions of vaccines' efficacy and knowledge about antibiotics.

\section{Conclusions:}

Perceptions of the risk of side-effects from vaccination and their understanding of the role of antibiotics for treating different infections are associated with one other within the general UK public. 
Research is needed to understand the nature of this association and identify areas of public understanding that are not exclusive to specific health interventions but that may be targeted to improve responsiveness to vaccine- and antibiotic-related public health interventions.

\section{Summaries:}

What is already known on this topic:

Antimicrobial resistance and vaccine hesitancy are biomedically connected public health challenges requiring differing public health messages for mitigation: avoid unnecessary antimicrobial consumption, and maximise vaccine uptake. One study, in Austria, has shown that patients wellinformed on the topic of antibiotic use had a higher likelihood of being vaccinated than poorlyinformed patients. Whilst public attitudes regarding antibiotics and vaccination are well-researched independently from one another in the UK, examination of the social connection between these issues has been neglected.

\section{What this study adds:}

This study demonstrates with a high-quality survey sample in the UK that incorrect knowledge regarding the use of antibiotics for treating different types of infection is strongly associated with negative perceptions of the risk of side-effects from vaccination, but not clearly associated with perceptions of the efficacy of vaccines. This finding prompts social researchers to engage with the nature of this connection to understand how the underlying public perceptions relating to these different public health issues can be addressed to improve antibiotic stewardship and vaccine uptake.

\section{Introduction:}

Vaccine hesitancy and antimicrobial resistance undermine many aspects of modern healthcare infrastructure. Since antimicrobial medicines have become ubiquitous within healthcare systems since their discovery and subsequent mass-production ${ }^{1,2}$ antimicrobial resistance has been described as an example of an infrastructural inversion rendering the otherwise invisible infrastructure of antimicrobial medication acutely visible. ${ }^{3}$ Recent viral crises such as the ongoing SARS-CoV-2 (COVID-19) pandemic have also made visible the significance of vaccination as a key part of public health infrastructure, as populations have been mandated to take a variety of precautions peculiar to daily life in order to mitigate the crisis until suitable vaccines are developed. Vaccine coverage remains a central pillar for health services' capacity to absorb the additional pressure of seasonal diseases such as influenza, for which antibiotics may be inappropriately prescribed. Antimicrobial resistance and vaccine hesitancy both feature in the World Health Organisation's top ten threats to global health. ${ }^{4}$

Antimicrobial resistance and vaccine uptake are biomedically connected issues. Vaccines are one among a number of interventions that have been proposed to address the development of antimicrobial resistance.$^{5-9}$ Vaccines can directly immunise against bacterial pathogens for example, reducing the use of antibiotics and consequent resistance selection pressure with the outcome of reducing levels of resistance in vaccine-addressed serotypes. ${ }^{10-14}$ Vaccines also immunise against viral pathogens for which antibiotics can be erroneously prescribed, or that may co-infect with bacterial pathogens causing secondary infections such as pneumonia or otitis media. ${ }^{7-9,15}$ With some international heterogeneity, the use of antibiotics to treat bacterial co-infections in COVID-19 patients has exemplified this use of antibiotics, with significant amounts of antibiotics being used to treat these cases and concerns being raised over the implications for antimicrobial resistance. ${ }^{16}$ In all of the above scenarios antibiotic use is minimised to society's benefit in the context of resistance development and 
individual benefit in terms of the negative effects of antibiotic use on the microbiome (for example, reduced diversity) and 'bystander' members of human microbial flora., ${ }^{77}$

The extent to which attitudes towards antimicrobial use and vaccine hesitancy are socially independent has not received significant attention despite the well-evidenced biomedical connection between vaccination and antimicrobial use. Public messaging around these interventions are significantly different: antimicrobial use is to be minimised, whilst vaccine uptake is to be maximised. This stark difference is reflective of the economic model for antimicrobials and vaccines as promoting vaccine uptake is clearly aligned with the interests of vaccine producers whilst the antimicrobial pipeline is hampered by the active minimisation of antimicrobials in practice. ${ }^{6}$ One study in Austria that has examined the correlation between influenza vaccine uptake and patients' knowledge about antibiotics found that patients who were better informed about the role of antibiotics had a significantly higher likelihood of being vaccinated..$^{18}$ The study's authors argue that a viable strategy to improve rates of influenza vaccination would be for physicians to counsel patients concerning antibiotics, influenza vaccination, and misconceptions about respiratory infections at the time of suggested vaccination. ${ }^{18}$ To date, this author has found no equivalent studies in the UK.

This study set out to examine whether some vaccine-related attitudes are associated in the UK with knowledge about antibiotics, independent of social and demographic factors previously demonstrated to be associated knowledge about antibiotics, using data from the Wellcome Monitor Wave 4. The findings of the study suggest that there are indeed be underlying attitudes, experiences, or perceptions common to the understandings of the risk of side-effects from vaccination and understandings of the role of antibiotics for treating infections. Directions for future research beyond the limitations of social survey methods to examine the nature and extent of these connections are discussed.

\section{Methods:}

\section{Data source}

The data used in this study are from the Wellcome Monitor Wave 4. ${ }^{19}$ The Wellcome Monitor is a survey that provides a barometer on the public's changing attitudes towards science and research in Britain. Specific to this wave, respondents were asked about both their knowledge regarding antibiotics and their attitudes towards vaccines.

The Wave 4 survey was conducted by the NatCen Social Research (NatCen) on behalf of the Wellcome Trust, and the survey data is available for reuse..$^{20}$ Respondents for the survey were recruited from the random-probability NatCen panel, which is a panel recruited from the British Social Attitudes (BSA) survey that uses a random-probability design and face-to-face survey mode. ${ }^{21}$ Fieldwork used a sequential mixed-mode design and was conducted from $16^{\text {th }}$ November to $16^{\text {th }}$ December 2018. Participants were invited to take part online and were contacted by telephone if they had not completed the survey after two weeks. A total of 2,708 respondents took part in the survey, with 2,336 (86\%) completing the survey online and $372(14 \%)$ completing the survey by telephone. ${ }^{21}$ Survey weights provided with the dataset adjust for nonresponse at three stage: the BSA, point of recruitment to the NatCen panel, and to the Monitor survey itself. ${ }^{21}$ These weights are applied in the estimates and hypothesis tests conducted for this study.

\section{Antibiotic- and vaccine-attitude measures}

The dependent variable in this study was survey respondents' level of knowledge about antibiotics, with vaccine-attitude variables as independent variables. 
The variable accounting for knowledge about antibiotics was constructed similarly to that used in a previous study which used Wave 3 data. ${ }^{22}$ The variable was derived from a question ("ABTreat" in the Wave 4 questionnaire ${ }^{23}$ ) that asked interviewees which of a list of conditions they believed could be effectively treated by antibiotics. To construct an ordered scale that ascends from best towards worse knowledge, each incorrect response to the question (for example, viral infections or fungal infections) was added together and the correct response (bacterial infections) was subtracted. For example, as neither viral infections or fungal infections can be treated effectively by antibiotics, individuals who chose those answers together were given two points. Likewise, for respondents who correctly identified that bacterial infections can be treated by antibiotics, their knowledge score was reduced by one. This process created a score range between -1 and 5. Only one respondent out of 2,708 provided enough incorrect responses to achieve a score of 5 , and so this respondent was included with the respondents who scored 4 to make a category of ' $4+$ '. The distribution of scores are shown in Table 1 and visualised in Figure 1, with $42.4 \%$ of the respondents achieving a perfect score of -1 .

Table 1 Weighted distribution of scores used for outcome variable.

\begin{tabular}{|l|l|l|}
\hline Antibiotic Knowledge Score & $\begin{array}{l}\text { Weighted Number of } \\
\text { Respondents }\end{array}$ & $\begin{array}{l}\text { Percentage of Weighted } \\
\text { Respondents }\end{array}$ \\
\hline-1 (Perfect response) & 1147 & 42.4 \\
\hline 0 & 641 & 23.7 \\
\hline 1 & 575 & 21.2 \\
\hline 2 & 202 & 7.5 \\
\hline 3 & 94 & 3.5 \\
\hline $4+$ & 49 & 1.8 \\
\hline
\end{tabular}

Weighted Distribution of Key Variables

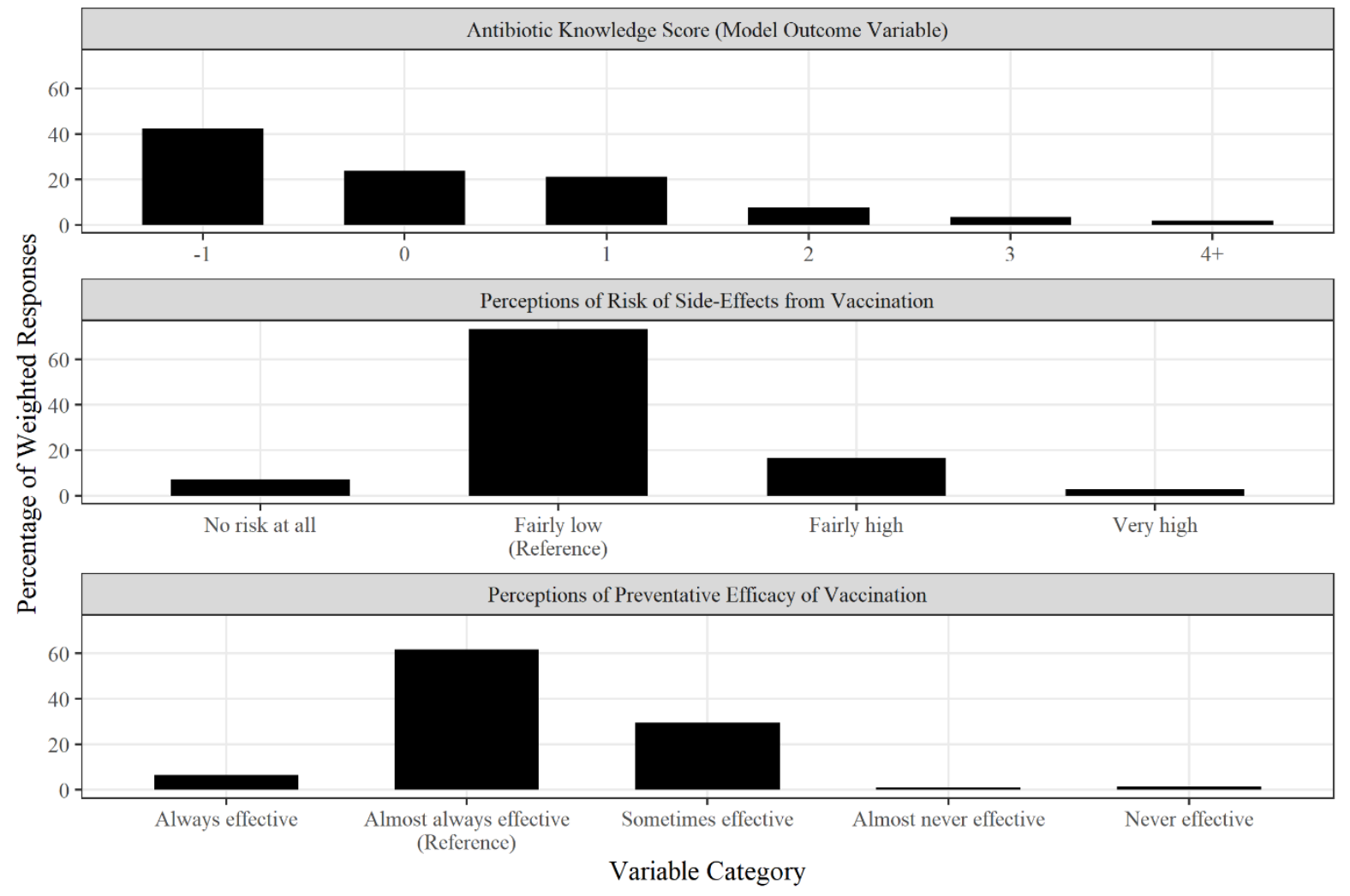

Figure 1 Weighted distribution of key antibiotic-and vaccine-related variables. 
Two vaccine-attitude variables were selected for analysis. The first variable was based on a question ("RiskVacc" in the Wave 4 questionnaire ${ }^{23}$ ) asking respondents to rate how high they believed the risks of serious side-effects are from vaccination. This question had four response categories, illustrated in Table 2 and Figure 1, and the modal category was used as the reference category for the purposes of the regression analysis. Over three quarters of respondents perceived the risk of side effects from vaccination to be 'fairly low', with a small minority (2.9\%) perceiving the risk to be 'very high'. The modal category was chosen as the reference rather than an extreme category in order to ascertain the difference between extreme groups and the norm as opposed to the difference of extreme groups from each other.

Table 2 Weighted distribution of responses regarding the perception of the risk of side-effects from vaccines.

\begin{tabular}{|l|l|l|}
\hline Response Category & $\begin{array}{l}\text { Weighted Number of } \\
\text { Respondents }\end{array}$ & $\begin{array}{l}\text { Percentage of Weighted } \\
\text { Respondents }\end{array}$ \\
\hline No risk at all & 188 & 7.0 \\
\hline Fairly low & 1977 & 73.5 \\
\hline Fairly high & 447 & 16.6 \\
\hline Very high & 76 & 2.9 \\
\hline
\end{tabular}

The second vaccine-attitude variable was based on a question ("PrevVacc" in the Wave 4 questionnaire) that asked respondents how often they felt that vaccines are effective at preventing diseases. This question had five response categories, illustrated in Table 3 and Figure 1, and the modal category was again used as the reference category for the purposes of the regression analysis. While the majority perceived a fairly low risk of side-effects from vaccination, there was not the same level of confidence in the effectiveness of vaccines, with over a quarter of respondents responding that vaccines were 'sometimes effective'.

Table 3 Weighted distribution of responses regarding the efficacy of vaccines as a preventative treatment.

\begin{tabular}{|l|l|l|}
\hline Response Category & $\begin{array}{l}\text { Weighted Number of } \\
\text { Respondents }\end{array}$ & $\begin{array}{l}\text { Percentage of Weighted } \\
\text { Respondents }\end{array}$ \\
\hline Always effective & 175 & 6.5 \\
\hline Almost always effective & 1662 & 61.6 \\
\hline Sometimes effective & 798 & 29.6 \\
\hline Almost never effective & 25 & 0.9 \\
\hline Never effective & 37 & 1.3 \\
\hline
\end{tabular}

These variables do not measure the totality of vaccine hesitancy or anti-vaccination sentiment, nor do they specifically distinguish between different types of vaccine (for example, between MMR and influenza). These variables are broad measures of perception regarding the risk of side-effects from vaccines, and the efficacy of vaccines as a preventative treatment. This apparent reduction of vaccine hesitant people to a singular public ${ }^{24,25}$ is a limitation of the data and method, which will be returned to in the Discussion.

\section{Statistical analysis}

The statistical significance level was $95 \%$ for all hypothesis tests in this study. An initial weighted $\chi^{2}$ test for independence was used to examine whether the two vaccine-attitude variables were distributed independently from the antibiotic knowledge outcome variable. Both tests provided evidence to reject the null hypotheses that either perceptions of the risk of side-effects resulting from vaccines $\left(\chi^{2}=191.8, \mathrm{df}=15, \mathrm{p}<0.001\right)$ or responses regarding the efficacy of vaccines as a preventative treatment $\left(\chi^{2}=185.8, \mathrm{df}=20, \mathrm{p}<0.001\right)$ were distributed independently from levels of knowledge about antibiotics. Following these results, regression analysis was used to examine the extent to which these 
relationships are sustained independently when controlling for known demographic, geographic, and attitudinal associations.

An ordinal logistic regression model was fitted, with the antibiotic knowledge score as the outcome variable. Explanatory variables were included alongside the vaccine-attitude measures to control for known associations with knowledge about antibiotics. The selection of explanatory variables to control in the model was informed by recent UK-based studies that employed similar randomprobability survey samples, including the preceding Wellcome Monitor Wave 3, to examine factors associated with knowledge and attitudes regarding antibiotics. ${ }^{22,26}$ Age, gender, level of education, employment status, and the consumption of online health information have been found to be associated with knowledge about the efficacy of antibiotics for treating bacterial and other pathogenic infections. ${ }^{22}$ A study that used an exploratory model-averaging approach to analyse two combined waves of the UK segment of the Eurobarometer survey additionally identified local and regional geographic factors and political attitudes associated with attitudes towards antibiotic prescription compliance. ${ }^{26}$ Both of these studies identified independent cross-sectional associations between knowledge about antibiotics and reported behaviours or attitudes regarding antibiotic prescription compliance. $^{22,26}$

Following the evidence of these studies, the model fitted in this study contained similar demographic, geographic, and attitudinal variables in order to isolate the independent relationship between the chosen vaccine-attitude variables and the antibiotic-related outcome variable. A number of studies have demonstrated that online sources and social media are increasingly important spaces in which information about antibiotics, antibiotic resistance, and vaccination are being disseminated and consumed. ${ }^{27-30}$ Taking into account the use of popular platforms and formats within the general category of 'the Internet', and the benefit of a large survey sample, information-source variables were included that distinguish between websites, social media platforms, and podcasts rather than simply referring to the Internet homogeneously. The variables included in the model contained a combined 54 missing observations, leaving a final sample of 2,654 analysed by the regression model.

Survey mode effects have been found associated with outcome variables on the topic of antibiotics. ${ }^{26}$ As the dataset used in this study was collected using online and telephone survey modes, a weighted $\chi^{2}$ test was used to test the independence of survey mode from the antibiotic knowledge outcome variable. The test $\left(\chi^{2}=130.5, \mathrm{df}=5, \mathrm{p}<0.001\right)$ provided evidence to reject the null hypothesis that survey mode and knowledge outcome were independent. Survey mode was consequently included in the regression model as a control variable. 


\section{Results}

Table 4 contains the results of the regression model used in this study with odds ratios (ORs) and 95\% confidence limits (CLs). Confidence limits that do not include 1 are considered statistically significant.

Table 4 Regression model results, asterisk denotes significant OR.

\begin{tabular}{|c|c|c|c|}
\hline Variable & Odds Ratio & $2.5 \%$ & $97.5 \%$ \\
\hline \multicolumn{4}{|c|}{ Age * Sex (Reference: $18-29$ Female) } \\
\hline 30-39 Female & $0.47 *$ & 0.33 & 0.68 \\
\hline 40-49 Female & $0.29 *$ & 0.20 & 0.42 \\
\hline 50-59 Female & $0.39 *$ & 0.27 & 0.57 \\
\hline 60-69 Female & $0.23 *$ & 0.15 & 0.36 \\
\hline 70+ Female & $0.32 *$ & 0.20 & 0.50 \\
\hline 18-29 Male & $1.53 *$ & 1.06 & 2.20 \\
\hline 30-39 Male & 1.46 & 0.88 & 2.42 \\
\hline 40-49 Male & $2.11 *$ & 1.26 & 3.54 \\
\hline 59-59 Male & 1.14 & 0.69 & 1.88 \\
\hline 60-69 Male & $1.73^{*}$ & 1.02 & 2.96 \\
\hline 70+ Male & 1.31 & 0.78 & 2.20 \\
\hline \multicolumn{4}{|c|}{ Education (Reference: Below Level 3) } \\
\hline No Qualifications & $1.63^{*}$ & 1.26 & 2.10 \\
\hline Level 3 & 0.87 & 0.69 & 1.11 \\
\hline Degree and Above & $0.59 *$ & 0.47 & 0.73 \\
\hline Other & 1.03 & 0.76 & 1.40 \\
\hline \multicolumn{4}{|c|}{ Employment Type (Reference: Paid Work) } \\
\hline Full-time Education & 0.83 & 0.57 & 1.20 \\
\hline Unemployed & 1.33 & 0.89 & 1.98 \\
\hline Retired & 1.20 & 0.88 & 1.62 \\
\hline Other Employment Type & 1.00 & 0.81 & 1.24 \\
\hline \multicolumn{4}{|c|}{ Rural/Urban Area (Reference: Rural) } \\
\hline Urban & $1.26^{*}$ & 1.03 & 1.53 \\
\hline \multicolumn{4}{|c|}{ Government Office Region (Reference: East Midlands) } \\
\hline East of England & 0.74 & 0.53 & 1.04 \\
\hline London & 1.11 & 0.80 & 1.54 \\
\hline North East & 1.06 & 0.68 & 1.63 \\
\hline North West & 0.75 & 0.54 & 1.06 \\
\hline Scotland & 1.17 & 0.82 & 1.66 \\
\hline South East & 0.85 & 0.61 & 1.18 \\
\hline South West & $0.69 *$ & 0.48 & 0.98 \\
\hline Wales & 0.98 & 0.64 & 1.49 \\
\hline West Midlands & 0.79 & 0.55 & 1.13 \\
\hline Yorkshire and the Humber & 0.89 & 0.62 & 1.27 \\
\hline \multicolumn{4}{|c|}{ Frequency of Health Information Seeking (Reference: Never) } \\
\hline Less often than once per month & $1.61 *$ & 1.16 & 2.23 \\
\hline Once or twice a month & 1.40 & 0.99 & 1.98 \\
\hline
\end{tabular}




\begin{tabular}{|c|c|c|c|}
\hline Once or twice a week & 1.40 & 0.96 & 2.02 \\
\hline On most days & 0.98 & 0.62 & 1.53 \\
\hline Several times a day & 1.14 & 0.60 & 2.14 \\
\hline \multicolumn{4}{|c|}{ Use of Information Sources for Health Information (Reference: Not used) } \\
\hline Websites & $0.76^{*}$ & 0.63 & 0.92 \\
\hline Podcasts & 0.95 & 0.68 & 1.32 \\
\hline Social Media & 1.05 & 0.89 & 1.25 \\
\hline \multicolumn{4}{|c|}{$\begin{array}{l}\text { How much of an impact can the actions of individual people have on drug resistant infections? } \\
\text { (Reference: A lot of impact) }\end{array}$} \\
\hline No impact at all & $2.63 *$ & 1.91 & 3.62 \\
\hline A little impact & $1.60^{*}$ & 1.33 & 1.93 \\
\hline Don't Know & $1.97 *$ & 1.45 & 2.67 \\
\hline Self-Report Low Understanding of the Topic & $2.64 *$ & 2.09 & 3.33 \\
\hline \multicolumn{4}{|c|}{ Risk of serious side-effects from vaccination (Reference: Fairly low) } \\
\hline Very high & $3.34 *$ & 2.13 & 5.25 \\
\hline Fairly high & $1.55^{*}$ & 1.26 & 1.90 \\
\hline No risk at all & $0.64 *$ & 0.46 & 0.87 \\
\hline \multicolumn{4}{|c|}{ Efficacy of vaccines for preventing disease (Reference: Almost always effective) } \\
\hline Never effective & 1.22 & 0.65 & 2.27 \\
\hline Almost never effective & 1.84 & 0.88 & 3.86 \\
\hline Sometimes effective & $1.25^{*}$ & 1.05 & 1.49 \\
\hline Always effective & 1.06 & 0.77 & 1.45 \\
\hline \multicolumn{4}{|l|}{ Interview Mode (Reference: Web) } \\
\hline Phone & $2.08 *$ & 1.69 & 2.56 \\
\hline
\end{tabular}

\section{Vaccine Attitudes}

The model provides evidence that respondents' attitudes towards vaccines were associated with their knowledge about antibiotics. Vaccine attitudes were divided into perceptions of risk of side-effects and perceptions of efficacy, and there was a clearer pattern to the relationship between perceptions of risk and understanding of antibiotics than between perceptions of efficacy and understanding of antibiotics.

Compared to the modal risk-perception category 'Fairly low', respondents who felt that vaccination posed no risk at all of side-effects $(\mathrm{OR}=0.64, \mathrm{CL}=0.46-0.87)$ were less likely to have poor knowledge about the role of antibiotics for treating different kinds of infections. Conversely, respondents who felt that vaccination posed fairly high $(\mathrm{OR}=1.55, \mathrm{CL}=1.26-1.90)$ or very high $(\mathrm{OR}=3.34, \mathrm{CL}=2.13-5.25)$ risks of side-effects were more likely to have poor knowledge about the utility of antibiotics. Figure 2 visualises these associations, showing a pattern in which more severe negative perceptions of the potential for vaccination to cause side-effects are associated with higher likelihoods of poor understandings regarding antibiotics. 


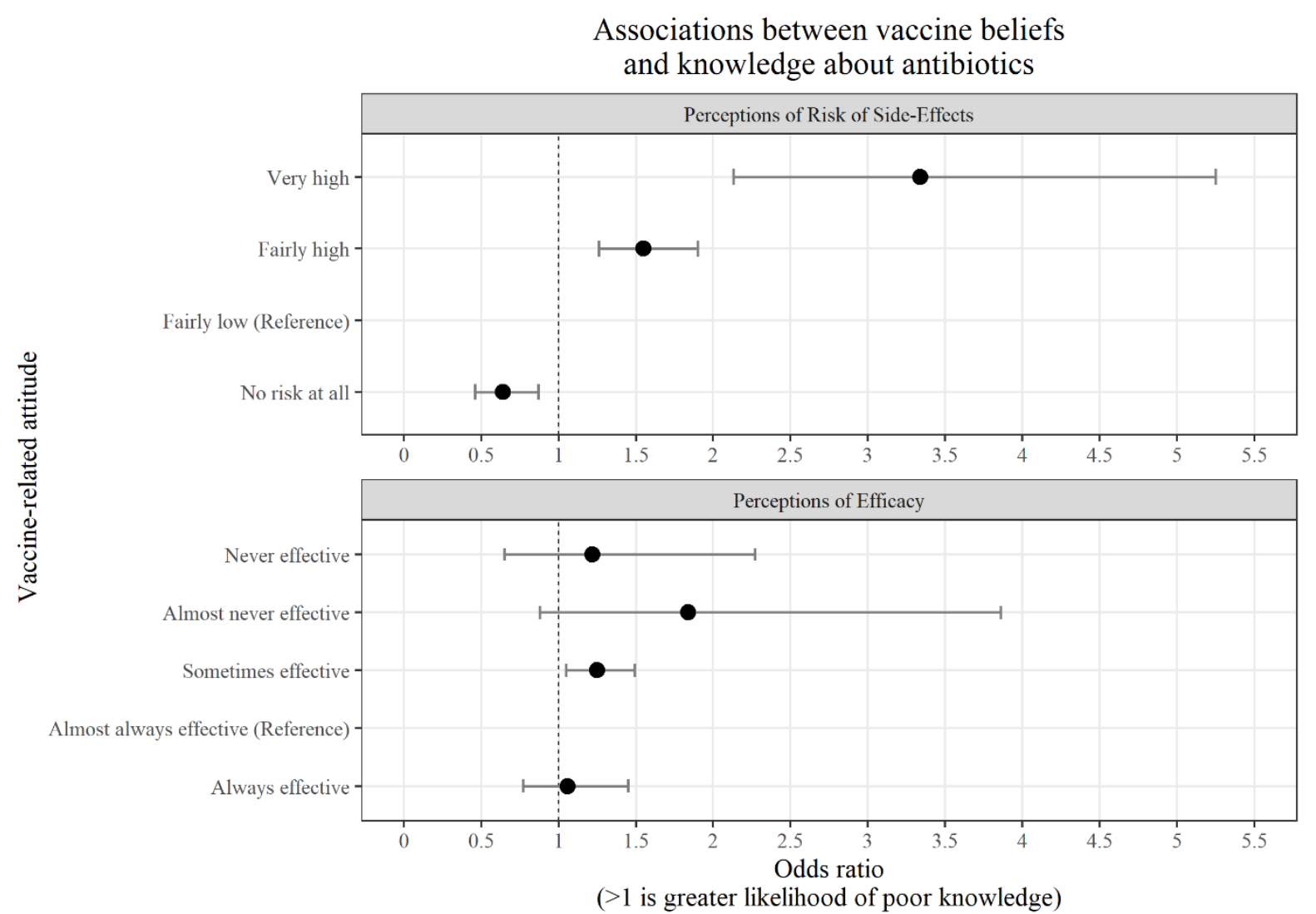

Figure 2 Odds ratio plot of independent associations between vaccine attitude responses and antibiotic knowledge score. OR greater than 1 indicates a higher likelihood of a poor knowledge score, and CLs not including the dotted line denote significance.

Associations between perceptions of the efficacy of vaccines for preventing disease and levels of knowledge about antibiotics present a different story. There were no significant differences evidenced in the model between the modal category 'Almost always effective' and extreme categories such as 'Always effective' $(\mathrm{OR}=1.06, \mathrm{CL}=0.77-1.45)$ or 'Never effective' $(\mathrm{OR}=1.22, \mathrm{CL}=0.65-2.27)$. The only statistically significant difference evidenced by the model was between the reference category respondents and respondents who felt that vaccines were 'Sometimes effective' $(\mathrm{OR}=1.25, \mathrm{CL}=1.05$ 1.49). These respondents were more likely to have poorer understanding of the use of antibiotics, though as illustrated in Figure 1 this difference between groups was not as large as exhibited by different perceptions of risks of side-effects.

Compared to the most commonly held perceptions of vaccines in these two areas, differences within the general public's perceptions of the risk of side-effects from vaccination are evidenced here as being associated more clearly with understandings of the use of antibiotics for treating different kinds of infections than are perceptions of the efficacy of vaccines as a preventative intervention. Members of the public who were more concerned about the risk of side-effects from vaccination were less likely to have a correct understanding of antibiotics as a treatment solely for bacterial infections rather than, for example, influenza or fungal infections. Concern over the side-effects of antibiotic medicines have been highlighted in previous research as being associated with a greater likelihood of antibiotic prescription incompliance. ${ }^{26}$ The evidence of this model could therefore be associated with perceptions of risk in relation to medicine consumption more broadly, however the nature of this connection would require specific qualitative follow-up research because 'risk' is a complex concept and its relation to vaccine uptake is not straightforward. ${ }^{24,25,31-33}$ Lawrence et al. for example identify separate publics of vaccine concern that coalesce around the safety and efficacy of vaccines, separating them into anti-vaccinationists who actively subvert vaccination practice and vaccine 
hesitant people who express discomfort about vaccines but do not refute vaccination as a practice. ${ }^{25}$ The available survey data does not distinguish between anti-vaccinationism and vaccine hesitancy in principle, but does suggest that members of the public who have more significant concerns about the risk of side-effects from vaccination are more likely to have misperceptions about the role of antibiotics in treating types of infections.

\section{Information-seeking and individual agency}

The only information source that presented an independent significant association in the model was the use of websites $(\mathrm{OR}=0.76, \mathrm{CL}=0.63-0.92)$ to look up health information. Neither podcasts $(\mathrm{OR}=0.95, \mathrm{CL}=0.68-1.32)$ nor social media $(\mathrm{OR}=1.05, \mathrm{CL}=0.89-1.25)$ were significantly associated with having better or worse knowledge about antibiotics. This evidence adds nuance to previous findings of positive associations between the use of the Internet as a source of medical research information and levels of knowledge about antibiotics using Wellcome Monitor Wave 3 data, ${ }^{22}$ and between trust in official health websites and compliance with antibiotic prescriptions but not between trust in personal health blogs and prescription compliance using Eurobarometer data. ${ }^{26}$ Respondents that used websites for health information were more likely in this study to have better knowledge about antibiotics compared to respondents who did not, whilst there was no evidenced direction of association regarding respondents who did or did not report using podcasts or social media as information sources.

All categories of the variable measuring respondents' perceptions of their own individual agency in addressing the issue of antimicrobial resistance presented significant differences from the reference category of respondents who felt that they could individually make 'A lot of difference' to antimicrobial resistance. Respondents who felt that they could only make a 'Little impact' $(\mathrm{OR}=1.60$, $\mathrm{CL}=1.33-1.93)$ or 'No impact at all' $(\mathrm{OR}=2.63, \mathrm{CL}=1.91-3.62)$ were more likely to have poor knowledge about what antibiotics should be used for. Individuals who responded that they did not know whether they could have an impact $(\mathrm{OR}=1.97, \mathrm{CL}=1.45-2.67)$, or felt that they had a low understanding of the issue ( $\mathrm{OR}=2.64, \mathrm{CL}=2.09-3.33)$, were also more likely to have poor knowledge about what antibiotics should be used for. A previous study found that respondents who felt that antibiotic resistance should be addressed at a level other than the individual or family level were almost twice as likely to have incompliant attitudes towards antibiotic prescriptions than respondents who felt that antibiotic resistance should be addressed by individual members of the public. ${ }^{26}$ The evidence of the current study specifies that perceptions of minimal individual impact upon the problem of antimicrobial resistance is also associated with lower levels of knowledge about the role of antibiotics in addressing different kinds of infections. Additionally, members of the public who feel uninformed about the issue of antimicrobial resistance perhaps unsurprisingly also tended to score lower on the knowledge outcome variable.

\section{Demographic and geographic associations}

There were a number of statistically significant demographic associations presented in the model. Age and sex were interacted, and there were associations between both age and sex and the outcome knowledge variable. Contrasted with 18-29 year old female respondents, all older categories of female respondents were less likely to have a poor score on the knowledge outcome variable. Conversely, 1829 year old male respondents $(\mathrm{OR}=1.53, \mathrm{CL}=1.06-2.20)$ were more likely to have a worse score on the outcome variable than 18-29 year old female respondents, with 40-49 year old male respondents $(\mathrm{OR}=2.11, \mathrm{CL}=1.26-3.54)$ and 60-69 year old male respondents $(\mathrm{OR}=1.73, \mathrm{CL}=1.02-2.96)$ also more likely to have worse antibiotic knowledge as measured by the outcome variable. There were no associations between employment type and the outcome variable, but there were associations between 
educational categories and the outcome variable. Contrasted with the reference category of having education qualifications below level 3 (A-levels and level 3 NVQs are examples of level 3 qualifications), respondents with no qualifications $(\mathrm{OR}=1.63, \mathrm{CL}=1.26-2.10)$ were more likely to have an incorrect perception of the utility of antibiotics for treating various infections whilst respondents educated to degree level or above $(\mathrm{OR}=0.59, \mathrm{CL}=0.47-0.73)$. These results generally corroborate other recent studies, for example previous findings that young women are more likely to have a good understanding of antibiotics' uses than young $\operatorname{men}^{22}$, or in the context of prescription compliance that older people and women were more likely to comply with antibiotic prescription instructions. ${ }^{26}$

Where respondents lived was occasionally associated with their antibiotic-related knowledge in the model, independent from their demographic or attitudinal characteristics. Respondents in urban areas $(\mathrm{OR}=1.26, \mathrm{CL}=1.03-1.53)$ were more likely to have poorer knowledge about antibiotics, though this was a relatively small significant association. The only region of England, Wales, and Scotland that was significantly different from the median region in terms of antibiotic-knowledge scores (the East Midlands) was the South West of England ( $\mathrm{OR}=0.69, \mathrm{CL}=0.48-0.98)$ where respondents were less likely to have a poor knowledge score. This evidence presents a different image to that of the geography of incompliant attitudes towards antibiotic prescriptions presented at the same geographic level with the same reference region in a previous study. ${ }^{26}$ In that study, Eurobarometer respondents living in London and Scotland were found to be over twice as likely to report an incompliant attitude towards antibiotic prescriptions whilst respondents in the North East of England were less likely to report such attitudes. Those findings were independent of similar controls to those used in this study. Together, the findings of the current study and previous work ${ }^{26}$ suggest that there remains as-yet unexplained regional variation in knowledge and attitudes regarding antibiotics and that there are differences between this variation in the contexts of knowledge about antibiotics and attitudes towards their use.

\section{Survey-mode association}

Interview mode was included in the regression model following a $\chi^{2}$ test which provided evidence that the mode of interview and result on the antibiotic knowledge outcome variable were not distributed independently from one another. The regression model results in Table 4 provide evidence of an independent survey mode difference between online respondents and telephone respondents in which telephone respondents $(\mathrm{OR}=2.08, \mathrm{CL}=1.69-2.56)$ were around twice as likely to have provided incorrect responses regarding the utility of antibiotics.

In a previous study, uncooperative respondents were found to have been more likely to report incompliant attitudes towards antibiotic prescriptions. ${ }^{26}$ Together, this previous study with this study suggest that there are potentially significant survey mode effects present in social research on the topic of antibiotics and antibiotic use in the UK. As telephone was the follow-up mode in the data collection for the Wellcome Monitor dataset used in this study, the direction of this association in conjunction with the previous finding regarding uncooperative respondents could suggest that reluctant respondents are not only more likely to be incompliant but also less well-informed about antibiotics than respondents with greater enthusiasm to respondent to a survey. Social researchers using surveys to investigate attitudes and behaviours relating to antibiotics should be cognisant of the potential for their chosen survey mode(s) to affect their sample and the distribution of antibioticrelated outcomes of interest. 


\section{Discussion}

Vaccination is one of a number of interventions for addressing antimicrobial resistance, and the public health challenges of antimicrobial resistance and vaccine hesitancy are biomedically connected ${ }^{5-9}$ and high priorities for the WHO. ${ }^{4}$ Despite their well-evidenced biomedical connections, minimal research has examined the extent to which vaccine hesitancy and attitudes towards antibiotic consumption correlate in the general population. One previous study in Austria found that there was a positive association between being well-informed about antibiotic use and vaccine uptake. ${ }^{18}$ This study set out to examine whether such an association exists in the UK using data from the Wellcome Montior Wave 4 survey.

\section{Higher vaccine risk perceptions are associated with antibiotic-related misperceptions}

Perceptions of the risk of side-effects from vaccination and the efficacy of vaccines as a preventative health intervention were examined in this study for their association with knowledge about antibiotics. The study found that perceptions of the level of risk of side-effects from vaccination were independently associated with knowledge about the role of antibiotics for treating infections. There were less pronounced differences between groups with different perceptions of the efficacy of vaccines in terms of their knowledge about antibiotics. The perceptions of vaccine risk and efficacy were contrasted to the most commonly reported category in each case, therefore these results suggest that relative to the majority of the general public there are significant differences in public understanding of antibiotic use between members of the public who are variably concerned about the risk rather than the efficacy of vaccines. These differences are independent of a variety of factors including demographic and geographic factors, information-seeking behaviours, and interview mode.

There are limitations to the inferences that may be drawn from the survey data in this study. It cannot be said, for example, that all vaccine hesitant individuals are less-informed about the role of antibiotics. Vaccine hesitancy is a complex phenomena that involves not only perceptions of risk and efficacy, but also trust, personal experience, and conceptualisations of health that may not be biomedical. ${ }^{24,25,31-33}$ Additionally, public perceptions of vaccination vary between the diseases targeted by specific vaccines. Risk perceptions and hesitancy relating to the MMR vaccine, for example, have a different intensity to those concerning influenza or polio. ${ }^{25,30,33}$ The conclusions relating to vaccine attitudes here are therefore narrow, relating specifically to generalised perceptions of the risk of sideeffects from vaccination and the efficacy of vaccination as a preventative intervention.

Future research should examine perceptions of antibiotic use specifically in relation to attitudes towards vaccination, as both areas require careful public health communication in order to maximise their benefit for society. Societal benefit itself for example has not always been a feature of vaccinefocused communication, ${ }^{31}$ and antibiotic-focused communications have also been inconsistent on relaying the societal benefits of appropriate consumption. ${ }^{34}$ If the commonalities between vaccine-risk perceptions and understanding of antibiotics' use suggested in this study signify broader similarities between types of vaccine hesitancy and behaviour with antibiotics, a deeper understanding of how these phenomena relate to one another may have use in improving public health communication on these issues. Previous research has highlighted the importance of social and cultural context alongside personal experience for understanding specific attitudes towards vaccination, ${ }^{25,32}$ and similar contextual influences have been discussed in the context of antibiotic use. ${ }^{35-37}$ The future research indicated here should be cognisant of these influences, and consider medical and veterinary care contexts such as parental decision-making and veterinarian-client communication as well as broader dynamics within the general population regarding antibiotic use as well as vaccine decisions. 


\section{Perceptions of individual agency in addressing antimicrobial resistance are associated with knowledge about antibiotics}

Previous qualitative research has found that some members of the public feel that they cannot positively influence antimicrobial resistance,$^{38}$ and members of the public who felt that antimicrobial resistance should be addressed at a level other than the individual or family were to be quantitatively more likely to report incompliant attitudes towards antibiotic prescription instructions. ${ }^{26}$ This study provides additional evidence to this finding from a different dataset, suggesting that individuals who feel that they can have minimal impact on antimicrobial resistance are also likely to be less informed about the role of antibiotics in treating infections than individuals who feel that they can have an impact.

Whilst causal associations cannot be made using this data, this study does suggest that members of the UK public who have a poor understanding of the role of antibiotics as specifically treating bacterial infections are also more likely to feel that they can personally have minimal impact on antimicrobial resistance and to perceive vaccination as a high-risk intervention. This study does not provide evidence that addressing a deficit in public knowledge about the role of antibiotics may necessarily improve either of these areas, but rather demonstrates that the issues of poor knowledge about antibiotics and high perception of vaccine risk are correlated, and possibly connected, in the general population and that there is a further attitudinal issue regarding personal enfranchisement linked to these that previous research has associated with behaviour following antibiotic prescriptions.

\section{Limitations of this study}

There are some limitations to this study that have been touched on in the discussion of the results. Firstly, the nature of the survey questions and the data they provide do not allow different levels of vaccine hesitancy to be examined in detail. 'Risk' is not a singular concept in relation to vaccination as has been elaborated by multiple qualitative studies..$^{24,25,31,32}$ Risk is conceptualised in several ways by members of the public and varies by disease, type, and timing of vaccination along with other factors. Survey data, including those used in this study, flatten this variability. This prompts future research to interrogate this topic. This variability has been acknowledged in the discussion of this study, but speculation on the nature of the associations found has been deliberately limited.

Furthermore, the variables used in the study do not directly indicate vaccine uptake. Whilst the cited literature does connect perceptions of risk and efficacy with vaccine hesitancy, this was not something that was explicitly available in this study.

Secondly, knowledge about antibiotics is an imperfect proxy for behaviour. ${ }^{34}$ As with the vaccinerelated variables, the outcome variable used in this study was not a behaviour variable and so inferences about correlations between vaccine or antibiotic behaviour cannot be made directly from this study and should be addressed in future research. Thirdly, as the data are cross-sectional, causal inferences cannot be made with certainty.

\section{Conclusion}

This study suggests that there are underlying attitudes, experiences, or perceptions that connect lay understandings of the risk of side-effects from vaccination and understandings of the role of antibiotics for treating infections. Perceptions of risk relating to vaccination are one component of vaccine decision-making by members of the public, and messaging around the inappropriateness of antibiotics for treating viral infections such as influenza has been central to public education initiatives regarding community antibiotic use. More detailed qualitative research is needed to understand the nature of the connection suggested here, as it could identify areas of public 
understanding that are not exclusive to specific health interventions but that could potentially be targeted to improve public responsiveness in both areas. Future research can also improve upon the questions that this study prompts qualitatively and quantitatively by examining vaccine-related attitudes and behaviours in tandem with antibiotic-related attitudes and behaviours and vice versa.

\section{Funding}

This study has no received specific funding from any organisation. During the conduction of this study and production of this manuscript, AA was funded by an Economic and Social Research Council PhD studentship (grant number: ES/J50015X/1), and subsequently has been employed on an unrelated project funded by a Wellcome Trust Collaborative Award (grant number 205393). Neither funding source was involved in the study design, data interpretation, writing, or decision to produce this manuscript.

\section{Acknowledgements}

The author thanks Isabelle Bi, Nicholas Dorward, and Pru Hobson-West for their feedback on a draft of this manuscript.

\section{References}

1) Bud R. Penicillin: Triumph and Tragedy. Oxford: Oxford University Press; 2007.

2) Podolsky SH. The Antibiotic Era: Reform, Resistance, and the Pursuit of a Rational Therapeutics. Baltimore: John Hopkins University Press; 2015.

3) Chandler CIR. Current accounts of antimicrobial resistance: stabilisation, individualisation and antibiotics as infrastructure. Palgrave Communications. 2020; 5: 53.

4) Ten threats to global health in 2019 [Internet]. World Health Organisation. Available from: https://www.who.int/vietnam/news/feature-stories/detail/ten-threats-to-global-health-in-2019.

5) The Review on Antimicrobial Resistance. Tackling Drug-Resistant Infections Globally: Final Report and Recommentations. 2016 May. Available from: https://amrreview.org/sites/default/files/160525_Final\%20paper_with\%20cover.pdf.

6) Lipsitch M, Siber GR. How Can Vaccines Contribute to Solving the Antimicrobial Resistance Problem? mBio. 2016; 7(3), e00428-16.

7) Bloom DE, Black S, Salisbury D, Rappuoli R. Antimicrobial resistance and the role of vaccines. PNAS. 2018; 115(51): 12868-12871.

8) Jansen KU, Knirsch C, Anderson AS. The role of vaccines in preventing bacterial antimicrobial resistance. Nature Medicine. 2018; 24: 10-20.

9) Buchy P, Ascioglu S, Buisso Y, Datta S, Nissen M, Tambyah PA, Vong S. Impact of vaccines on antimicrobial resistance. Interational Journal of Infectious Diseases. 2020; 90: 188-196.

10) Farrell DJ, Klugman KP, Pichichero M. Increased Antimicrobial Resistance Among Nonvaccine Serotypes of Streptococcus pneumoniae in the Pediatric Population After the Introduction of 7Valent Pneumococcal Vaccine in the United States. The Pediatric Infectious Disease Journal. 2007; 26(2): 123-128.

11) Hampton LM, Farley MM, Schaffner W, Thomas A, Reingold A, Harrison LH, Lynfield R, Bennett NM, Petit S, Gershman K, Baumbach J, Beall B, Jorgensen J, Glennen A, Zell ER, Moore M. Prevention of antibiotic-nonsusceptible Streptococcus pneumoniae with conjugate vaccines. The Journal of Infectious Diseases. 2012; 205: 401-411.

12) Moore MR, Link-Gelles R, Schaffner W, Lynfield R, Holtzman C, Harrison LH, Zansky SM, Rosen JB, Reingold A, Scherzinger K, Thomas A, Guevara RE, Motala T, Eason J, Barnes M, Petit S, Farley MM, McGee L, Jorgensen JH, Whitney CG. Effectiveness of 13-valent pneumococcal conjugate vaccine for prevention of invasive pneumococcal disease in children in the USA: a matched case-control study. Lancet Respiratory Medicine. 2016; 4(5): 399-406.

13) Tomczyk S, Lynfield R, Schaffner W, Reingold A, Miller L, Petit S, Holtzman C, Zansky SM, Thomas A, Baumbach J, Harrison LH, Farley MM, Beall B, McGee L, Gierke R, Pondo T, Kim L. Prevention of Antibiotic-Nonsusceptible Invasive Pneumococcal Disease With the 13-Valent Pneomococcal Conjugate Vaccine. Clinical Infectious Diseases. 2016; 62(9): 1119-25. 
14) Andrews JR, Baker S, Marks F, Alsan M, Garrett D, Gellin BG, Saha SK, Qamar FN, Yousafzai MT, Bogoch II, Antillon M, Pitzer VE, Kim J-H, John J, Gauld J, Mogasale V, Ryan ET, Luby SP, Lo NC. Typhoid conjugate vaccines: a new tool in the fight against antimicrobial resistance. Lancet Infectious Diseases. 2019; 19: e26-e30.

15) Klein EY, Monteforte B, Gupta A, Jiang W, May L, Hsieh Y-H, Dugas A. The frequency of influenza and bacterial coinfection: a systematic review and meta-analysis. Influenza and Other Respiratory Viruses. 2016; 10(5): 394-403.

16) Nature Microbiology Editorial Board. Antimicrobial resistance in the age of COVID-19. Nature Microbiology. 2020; 5: 779.

17) Relman DA, Lipsitch M. Microbiome as a tool and a target in the effort to address antimicrobial resistance. PNAS. 2018; 115(51): 12902-12910.

18) Hoffman K, van Bijnen EME, George A, Kutalek R, Jirovsky E, Wojczewski S, Maier M. Associations between the prevalence of influenza vaccination and patient's knowledge about antibiotics. BMC Public Health. 2015; 15: 981.

19) Steen B, Jessop C, Greenwood E, Sturgis P, Perera P, Ross C, Clements L, Woodgate P, Bleasdale B, Hickman M, Rinvolucri M, Brasting T, Nazir F. Wellcome Monitor Report 2018. 2019 Nov. Available from: https://wellcome.ac.uk/sites/default/files/wellcome-monitor-2018report.pdf.

20) Wellcome Monitor Wave 4 Dataset [dataset]. 2019 Nov. Available from: https://wellcome.ac.uk/sites/default/files/wellcome-monitor-2018-dataset-data-dictionary.xlsx.

21) NatCen Social Research. Wellcome Monitor Wave 4 Appendix A: Detailed Methodology. 2019 Nov. Available from: https://wellcome.ac.uk/sites/default/files/wellcome-monitor-2018appendix-a-methodology.pdf.

22) Anderson A. Online health information and public knowledge, attitudes, and behaviours regarding antibiotics in the UK: Multiple regression analysis of Wellcome Monitor and Eurobarometer Data. PLoS ONE. 2018; 13(10): e0204878.

23) NatCen Social Research. Wellcome Monitor Wave 4 Appendix B: Questionnaire. 2019 Nov. Available from: https://wellcome.ac.uk/sites/default/files/wellcome-monitor-2018-appendix-bquestionnaire.pdf.

24) Hobson-West P. 'Trusting blindly can be the biggest risk of all': organised resistance to childhood vaccination in the UK. Sociology of Health and Illness. 2007; 29(2): 198-215.

25) Lawrence HW, Hausman BL, Dannenberg CJ. Reframing Medicine's Publics: The Local as a Public of Vaccine Refusal. Journal of Medical Humanities. 2014; 35: 111-129.

26) Anderson A. Analysing incompliant attitudes towards antibiotic prescription completion in the UK. Journal of Antimicrobial Chemotherapy. 2020; 75: 756-763.

27) Kendra RL, Karki S, Eickhold JL, Gandy L. Characterizing the Discussion of Antibiotics in the Twittersphere: What is the Bigger Picture? Journal of Medical Internet Research. 2015; 17(6): e154.

28) Dyar OJ, Castro-Sánchez E, Holmes AH. What makes people talk about antibiotics on social media? A retrospective analysis of Twitter use. Journal of Antimicrobial Chemotherapy. 2014; 69: 2568-2571.

29) Yaqub O, Castle-Clarke S, Sevdalis N, Chataway J. (2014) Attitudes to vaccination: A critical review. Social Science \& Medicine. 2014; 112: 1-11.

30) Campbell H, Edwards A, Letley L, Bedford H, Ramsay M, Yarwood J. Changing attitudes to childhood immunisation in English parents. Vaccine. 2017; 35: 2979-2985.

31) Hobson-West P. Understanding vaccination resistance: moving beyond risk. Health, Risk \& Society. 2003; 5(3): 273-283.

32) Hobson-West P. The Construction of Lay Resistance to Vaccination. In: Shaw I, Kauppinen K, editors. Constructions of Health and Illness: European Perspectives. Aldershot and Burlington: Ashgate; 2004. p. 89-106. 
33) Brown KF, Kroll JS, Hudson MH, Ramsay M, Green J, Long SJ, Vincent CA, Fraser G, Sevdalis N. Factors underlying parental decisions about combination childhood vaccinations including MMR: A systematic review. Vaccine. 2010; 28: 4235-4248.

34) Will CM. The problem and the productivity of ignorance: Public health campaigns on antibiotic stewardship. The Sociological Review. 2020; 68: 55-76.

35) Cabral C, Ingram J, Hay AD, Horwood J, on behalf of the TARGET team. "They just say everything's a virus" - Parent's judgement of the credibility of clinician communicaiton in parimary care consultations for respiratory tract infections in children: A qualitative study. Patient Education and Counseling. 2014; 95: 248-253.

36) Smith M, King C, Davis M, Dickson A, Park J, Smith F, Currie K, Flowers P. Pet owner and vet interactions: exploring the drivers of AMR. Antimicrobial Resistance and Infection Control. 2018; 7: 46.

37) Anderson A. "We tried to, but life gets in the way": The Value of Cognitive Interviewing for Testing a Questionnaire on Antibiotic Consumption Behaviours. Version 1. SocArXiv [Preprint]. [Posted 2019 Nov 1]: [25 p.]. Available from: https://osf.io/preprints/socarxiv/zyq2t/.

38) Hawkings NJ, Wood F, Butler CC. Public attitudes towards bacterial resistance: a qualitative study. Journal of Antimicrobial Chemotherapy. 2007; 59: 1155-1160. 